\title{
An attempt to delineate very low frequency electromagnetic signals associated with earthquakes
}

\author{
*Toshi Asada ${ }^{1}$, Hisatoshi Baba ${ }^{1}$, Mamoru Kawazoe ${ }^{2}$, and Masahisa Sugiura ${ }^{3}$ \\ ${ }^{1}$ Research Institute of Science and Technology, Tokai University, 1117 Kitakaname, Hiratsuka, Kanagawa 259-1292, Japan \\ ${ }^{2}$ School of Engineering II, Tokai University, 2-28 Tomigaya, Shibuya-ku, Tokyo 151-0063, Japan \\ ${ }^{3}$ Research Institute of Science and Technology, Tokai University, 2-28 Tomigaya, Shibuya-ku, Tokyo 151-0063, Japan
}

(Received April 13, 2000; Revised August 29, 2000; Accepted September 29, 2000)

\begin{abstract}
We report on our observation of pulse-like electromagnetic signals in the frequency range $1 \sim 10 \mathrm{kHz}$ that we associate with earthquakes. The severest difficulty in separating earthquake-associated VLF signals from those originating in lightning discharges stems from the circumstance that the latter signals are overwhelming in number compared with the former. While claims have often been made of observation of electromagnetic signals in association with earthquakes, most of the claims, excepting a few, heavily rely on temporal correlation. By means of simple instrumentation and data processing software, our method by and large enables us to isolate VLF signals whose direction of arrival is well focussed at the epicenter direction. In this preliminary report we present several examples that positively demonstrate the existence of a class of VLF signals having a peak frequency of occurrence $1 \sim 4$ days prior to earthquakes of Magnitude 4 6. With an accumulation of experience the technique described in this paper would seem to offer a promising approach towards earthquake prediction.
\end{abstract}

\section{Introduction}

Observations of electromagnetic signals have often been linked to earthquakes (e.g., Yoshino et al. (1985), Fujinawa and Takahashi (1990), and earlier references quoted in the monographs edited by Hayakawa and Fujinawa (1994) and Hayakawa (1999)). However, the purported linkages are, in the majority of cases, through temporal relationships between the observed electromagnetic signals and the occurrence of earthquakes. Other studies that incorporate detection of the direction of signal arrival, while constituting a substantial improvement, need more cases of observation before becoming operational (e.g., Yoshino et al., 1985).

There have been two cases of fortuitous detection of decametric electromagnetic radiation that is considered to be causally related each to a major earthquake. These observations were conducted with interferometers intended for radio astronomical purposes and not for seismological study; relevant papers, Warwick et al. (1982) and Maeda and Tokimasa (1996) are discussed in an appropriate context in Section 4.

The study we have conducted is sufficiently different in design from other similar observations. In this paper we give a preliminary report on the results of our effort to delineate a class of electromagnetic emissions of earthquake origin from those of other sources. Electromagnetic signals investigated in this study are in the very low frequency (VLF) range, in the vicinity of 1 to $10 \mathrm{kHz}$. Most of the electromagnetic signals of natural origin observed in this frequency range

*In alphabetical order.

Copy right $(\mathrm{C})$ The Society of Geomagnetism and Earth, Planetary and Space Science (SGEPSS); The Seismological Society of Japan; The Volcanological Society of Japan; The Geodetic Society of Japan; The Japanese Society for Planetary Sciences. are atmospherics (or sferics in short), that is, signals mainly from lightning discharges. Despite this unfavorable circumstance the art achieved by this study enables us to separate those minority signals originating in earthquakes from the overwhelmingly numerous incidences of sferics. A guiding concept underlying our technique is that signals observed at a location in association with an earthquake must come predominantly from a distinct direction, namely, that of the epicenter, while the signal sources of sferics are dispersed in azimuth when viewed from a single site.

\section{Instrumentation}

Our instrumentation for the detection of pulse-like magnetic field consists of two identical ferrite bar-coil aerials and channel pre-amplifiers. The two aerials are set parallel to two horizontal reference axes that are orthogonal to each other. Figure 1 depicts the antenna system installed on the roof of one of the buildings on the Tokai University Shonan Campus. The two aerials are oriented towards the north-south and east-west directions. The antenna gain is of dumbbell shape so that the direction of arrival of a signal can be determined from the two orthogonal components of the incident signal by elemental geometry, if it is assumed that the incident signal is plane-polarized in the plane orthogonal to the direction of propagation. In addition, our recording system is such that the waveform can readily be examined by plotting the digital data.

At present, observations are being made continuously at three sites, Shonan Campus (SHN), Shimizu (SMZ), and Kumamoto (KMM); locations of these sites are indicated with dark dots on the map shown in Fig. 2. In this paper we give results obtained at these stations for the following 


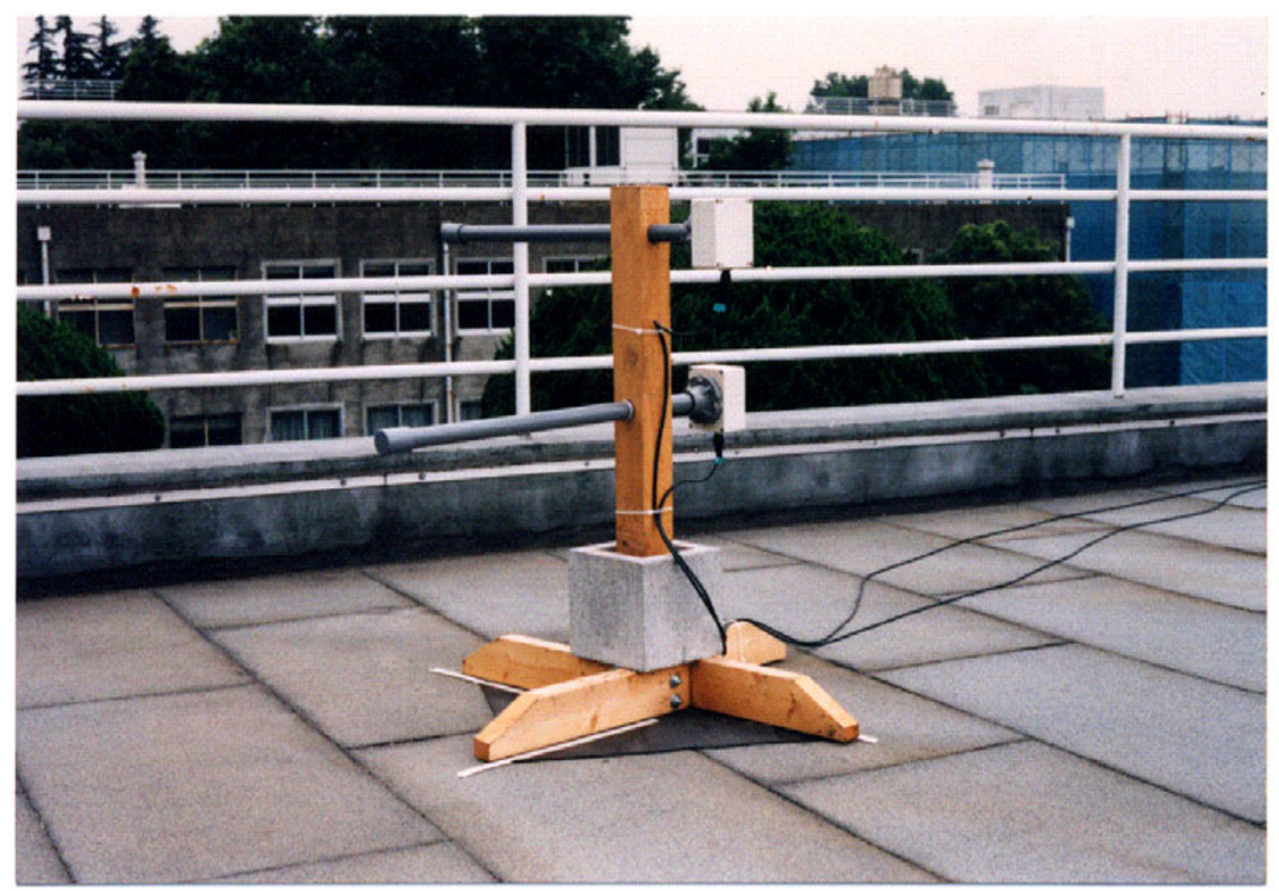

Fig. 1. Illustrating the antenna system on the roof of one of the buildings on Shonan Campus. Each antenna unit consists of a ferrite-coil antenna and a pre-amplifier. The two axes are oriented in the north-south and east-west directions.

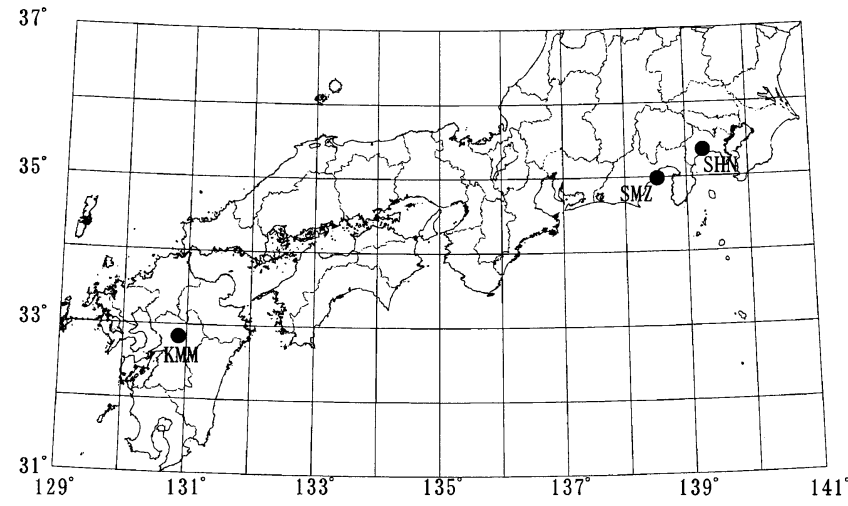

Fig. 2. Locations of the three stations, Shonan Campus (SHN), Shimizu (SMZ), and Kumamoto (KMM)

periods. For Shonan Campus: September 1996 to present and for Shimizu and Kumamoto: March 1997 to present.

Our algorism selects only those signals in which the two orthogonal components vary proportionately. This amounts to selecting incident pulses that are plane-polarized in the plane normal to some direction. This latter direction is designated as the direction of incidence. We do not ask the question of why the earthquake-related electromagnetic pulses are planepolarized. Rather, we adopt this polarization characteristic as a working hypothesis, and if the directions of incidence of the signals selected are well focussed in the direction of the epicenter, then we deduce that our selection rule is viable.

The software counts the cumulative number of events occurring in a unit time interval, and records for each event the azimuth of the direction of signal arrival. Thus the cumulative number is given as a function of azimuth. For the analysis presented in this paper the unit time is taken to be one day (i.e., 24 hours). This time interval can be of any length, and may be appropriately sampled if so desired, so long as the obtained results are statistically meaningful to achieve the intended objective.

The azimuth of signal calculated as described above has a $180^{\circ}$ ambiguity, that is, the system does not distinguish the direction of arrival, say, $\theta$ from $\theta \pm 180^{\circ}$. This ambiguity, however, presents no difficulty in the context of the discussions given in this paper, where the observed results on electromagnetic signals are compared with a set of seismic data already in existence. In any case, when observations are made at two or more sites, the ambiguity in the direction of signal arrival can be eliminated by means of triangulation.

It is remarked here that we make no claim that signals recorded are the only electromagnetic signals emitted by earthquakes. Setting criteria for the selection of signals is a difficult task, but in this study we have adopted a pragmatic approach with an underlying concept that any set of criteria that leads us to a useful result is a satisfactory set. Finding an optimum set of criteria is obviously an important task left for future study.

\section{Observation}

The primary purpose of this report is to demonstrate that our system of observation is capable of distinguishing electromagnetic signals originating in earthquakes from those coming from other sources. With this objective in mind we present four representative cases that typify our observation.

In presenting the occurrence frequency distribution of signal arrivals two different methods can be used. In one, plane Cartesian coordinates are used, in which the azimuth of the direction of signal arrival is represented by abscissa (along 


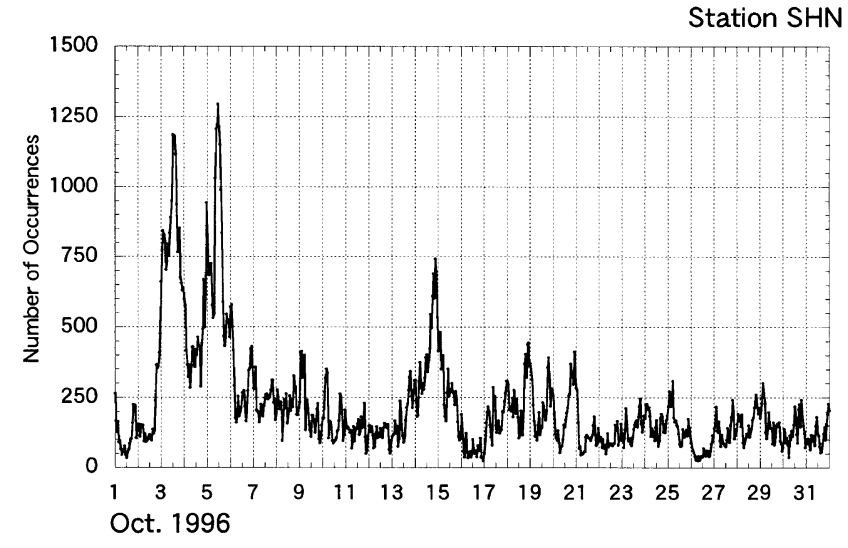

Fig. 3. The number of occurrences of VLF signals for each hour of October 1 through 31, 1996, integrated over all azimuth.

the horizontal $x$-axis) covering $180^{\circ}$ and the number of occurrences by ordinate (along the vertical $y$-axis). In another, polar coordinates are used, with the radial distance from the origin giving the number of occurrences, and with the polar angle indicating the azimuth of the signal arrival direction (the north and east directions being along the positive $y$ - and $x$-axis, respectively, of a Cartesian coordinate system). In this report the polar coordinate representation is mainly used to assist the visual perception of the signal arrival direction.

\subsection{Case I: October 5, 1996 earthquake}

The frequency of occurrence of VLF signals observed at Shonan Campus during the entire month of October 1996 is shown in Fig. 3 without regard to the direction of signal arrival. In the figure the vertical dotted lines correspond to the beginning of each day, i.e., 0 hour of the day indicated. In this paper, local time (JST) is used throughout. Upon inspection of Fig. 3 one immediately observes high VLF activity on the 3rd and 5th days of October. However, that most of the signals conspicuous in Fig. 3 for these two days are from sferics will become clear when we come to Fig. 4. Figure 3 shows the well-known feature in the diurnal variation of the occurrence frequency of VLF emissions from lightning discharges, namely a maximum near midnight. Remembering this feature has been found to be helpful in interpreting observation.

In Fig. 4, we present in the polar coordinate format the distribution of the frequency of occurrence of signal arrivals in the 24-hour period of each of the six days of October 1 through 6, 1996 obtained at Shonan Campus. There was an earthquake of Magnitude $(M)=4.4$ in Shizuoka Prefecture (at latitude $35.05^{\circ} \mathrm{N}$ and longitude $138.03^{\circ} \mathrm{E}$, at the depth of $26 \mathrm{~km}$ ) on October 5. On each of the six panels in Fig. 4, we have indicated the epicenter direction by a dotted line with an arrow at the tip. Special attention is called to the presence of a group of VLF emissions that is sharply bunched together around the epicenter direction. Such a group of emissions is most pronounced on October 3, panel (c), and is clearly identifiable on October 4 , (d), though smaller in number than on October 3. The presence of the same group of emissions is seen on Oct. 2, (b), and Oct. 5, (e), still with notable clarity. We consider these persistent emissions arriving from the epicenter direction as being VLF emissions causally related to the earthquake of October 5 .

The location of the epicenter of the October 5 earthquake is indicated by a black square in Fig. 5 (for Oct. 3). The distribution of signal arrival direction for October 3 is shown in Fig. 5, which is identical with panel (c) of Fig. 4 excepting that the polar graph is placed on a map with its origin coincident with the location of the observation site to see the situation in proper perspective. This figure shows the existence of a group of signals coming from the direction of the epicenter that are clearly separated from signals from other sources. These other sources presumably include lightning that took place in the general direction of northwest as reported by the Lightning Information Company of Franklin Japan. The locations of these lightning discharges are shown by dark dots scattered over the Sea of Japan roughly between the direction of Sado and the Noto Peninsula.

In Fig. 4, we see that after the peak on October 3 the VLF signals coming from the epicenter direction became gradually less pronounced toward the day of the earthquake, i.e., October 5. There is indication of the presence of a period of gradual build-up preceding the peak activity on October 3 and of a period of gradual decay after the peak. In summary, with the earthquake of October 5 there was a peak VLF activity two days prior to the day of the earthquake.

\subsection{Case II: March 26, 1997 earthquake}

At 17:31 JST on March 26, 1997 there was an earthquake of Magnitude 6.3 with the epicenter location at latitude $31.98^{\circ} \mathrm{N}$ and longitude $130.37^{\circ} \mathrm{E}$ and at a depth of $8.0 \mathrm{~km}$. In conjunction with this earthquake the Kumamoto station observed a large number of occurrences of VLF emission. Among them there was a group of emissions persistently coming from the epicenter direction. This VLF activity began on March 22 and appears to have lasted till March 26. The earthquake of March 26 differs from other cases dealt with in this paper in that it was accompanied by several aftershocks. Specifics (i.e., time of occurrence, magnitude M, and depth $d$ ) of the largest three of the aftershocks are as follows: 17:39 JST, $\mathrm{M}=4.7, d=8.0 \mathrm{~km} ; 18: 05 \mathrm{JST}, \mathrm{M}=4.5$, $d=10.0 \mathrm{~km} ; 22: 44 \mathrm{JST}, \mathrm{M}=11.0 \mathrm{~km}$. The locations of the epicenters of these aftershocks are only slightly shifted from that of the main shock.

At this stage of our investigation we do not know whether or not the aftershocks contributed any VLF emissions in addition to those from the main shock. Hence here we proceed regarding all the VLF emissions as coming from the epicenter of the main shock. If the aftershocks contributed any, the effects may well be taken care of by assuming an expanded source area. In terms of our present procedure this amounts to relaxing the directional requirement for a VLF emission to be considered as originating from the epicenter. In any case, it appears that ignoring the aftershocks would not present any fundamental difficulty in this discussion.

There is another caveat with Case II that cannot be ignored. That is, there was a continuing thunderstorm activity concurrent with the VLF emissions presumed to come from the earthquake. We cope with this problem by a contention that the incidence of the earthquake-related VLF emission is well focussed in the epicenter direction, while the lightning sources are more spread out rather than being focussed in a 


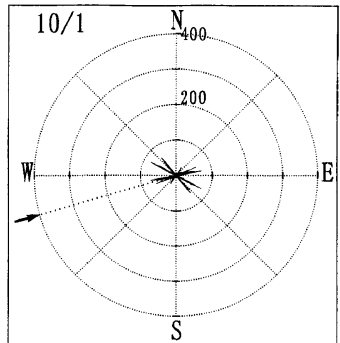

(a)

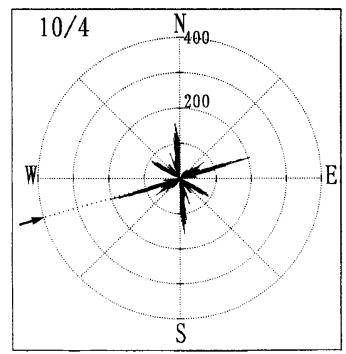

(d)

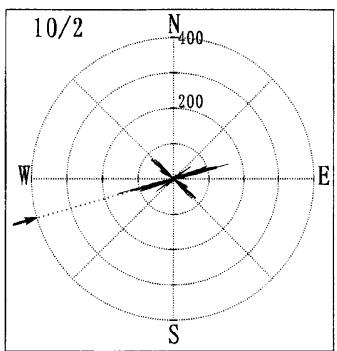

(b)

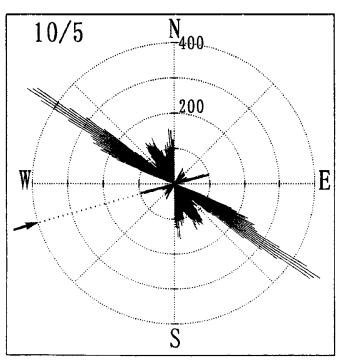

(e)

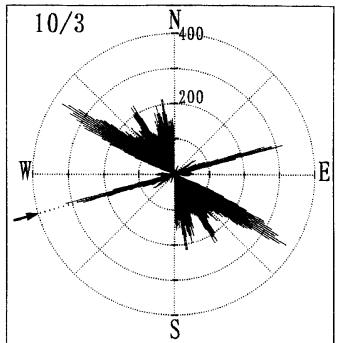

(c)

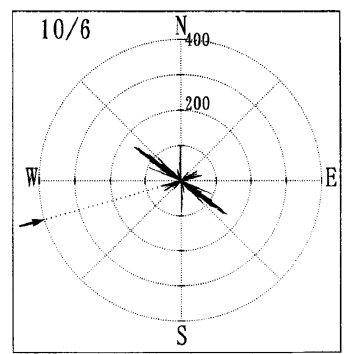

(f)

Fig. 4. The distribution of VLF signal arrival direction in 24 hours on each of six days, October 1 through 6, 1996 at Shonan Campus (Case I, an earthquake of $\mathrm{M}=4.4$ on Oct. 5). The radial distance represents the total number of occurrences. The epicenter direction is indicated by a dotted line with an arrow at the tip.

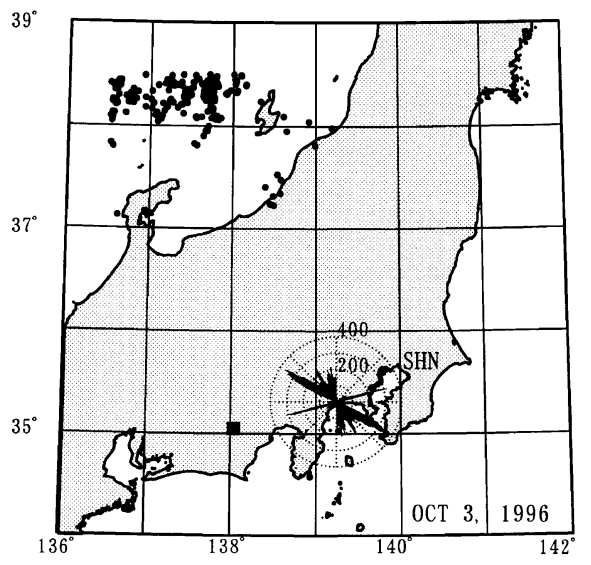

Fig. 5. Observation of VLF signals at Shonan Campus on October 3, 1996 (Case I, an earthquake of $\mathrm{M}=4.4$ on Oct. 5). The epicenter is indicated with the black square. The dots over the Sea of Japan show locations of lightning discharges (see the text).

single direction, and often shift in azimuth with time.

Panels (a), (b), .., (h) in Fig. 6 show in polar coordinate representation the distribution of the source directions of VLF radiation on March 20 through 27, 1997. The direction towards the epicenter is indicated on each panel by a dotted line marked with an arrow. We summarize our diagnosis of these figures: (i) No notable emissions from the epicenter area on Mar. 20. (ii) Little emission from the epicenter area, if any, is seen on Mar. 21. (iii) There is a group of emissions, large in number, bunched together towards the epicenter area on Mar. 22. (iv) The emission distribution is dominated by VLF emissions from a large number of sferics on Mar. 23. Much less frequent earthquake-related emissions exist in the shadow of sferics. (v) Occurrences of earthquake-related emission are numerous, and there are a relatively small number of sferics signals on Mar. 24. (vi) On Mar. 25 there is a group of emissions that can be considered to be from the earthquake. (vii) A small bunch of emissions is directed towards the epicenter on Mar. 26, but this may, or may not, have come from the earthquake. (viii) On Mar. 27, probably there were no earthquake-related emissions.

The distribution of the emission direction shown in panel (e) of Fig. 6, for March 24, is reproduced in geographical perspective in Fig. 7. Although not shown, there was no trace of emissions from the epicenter direction on March 28. Thus we conclude that for Case II, earthquake-related emissions were observed on March 22 through 26 with high emission rates on March 22 and 24, 4 and 2 days before the earthquake, respectively.

\subsection{Case III: March 9, 1999 earthquake}

The third case concerns the $M=4.5$ earthquake of March 9, 1999, which occurred at 12:53 JST near the Kumamoto station at a depth of $10 \mathrm{~km}$. The epicenter of this earthquake was at $32.95^{\circ} \mathrm{N}, 131.02^{\circ} \mathrm{E}$. For this case, sferics activity during the period of several days in the vicinity of March 9 was substantially lower than the comparable periods in the three other cases discussed in this paper. Panels (a), (b), .., (f) in Fig. 8 show the results for March 5, 6, ., 10, respectively. The direction towards the epicenter is indicated in each panel by a dotted line marked with an arrow. The following is a synopsis of our diagnosis of these diagrams: (i) There is an indication of earthquake-associated VLF emission on Mar. 5 (a). Though not shown, there are no VLF emissions from the direction of the epicenter on Mar. 4. (ii) Earthquake-related emissions fully developed on Mar. 6 (b) and maintained the level of activity on Mar. 7 (c). The results for Mar. 6 are reproduced in Fig. 9, showing the locations of the station and 


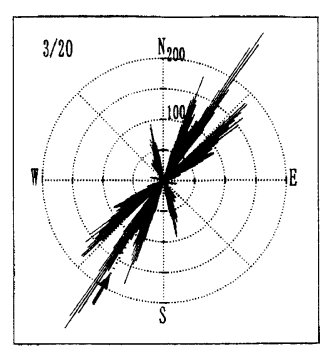

(a)

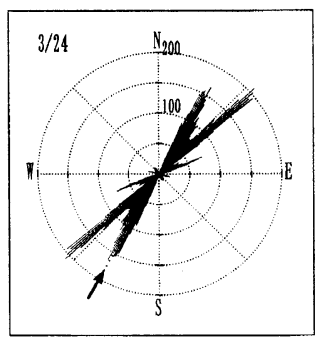

(e)

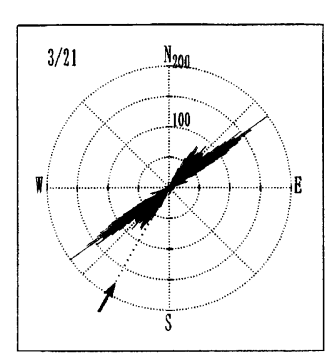

(b)

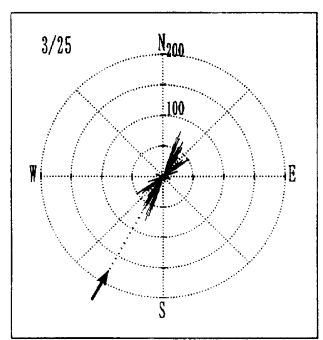

(f)

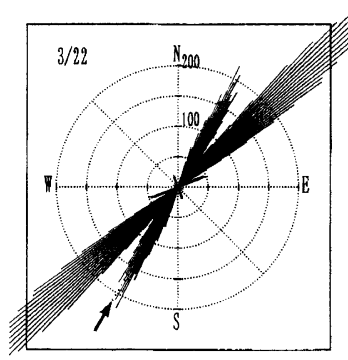

(c)

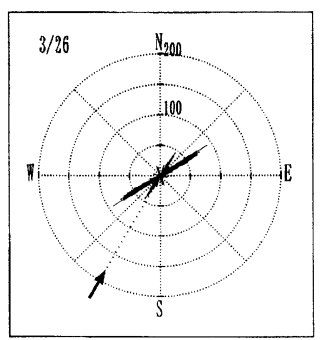

(g)

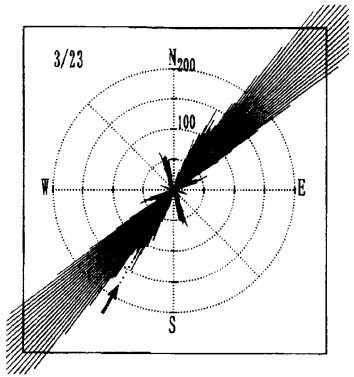

(d)

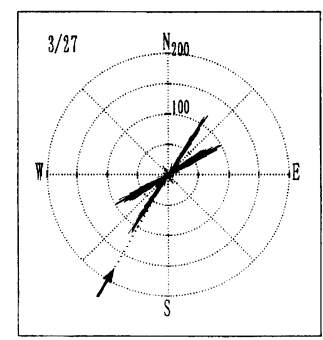

(h)

Fig. 6. The distribution of VLF signal arrival direction in 24 hours on each of the eight days, March 20 through 27, 1997, at Kumamoto (Case II, an earthquake of $\mathrm{M}=6.3$ on Mar. 26). The epicenter direction is shown by a dotted line with an arrow at the tip.

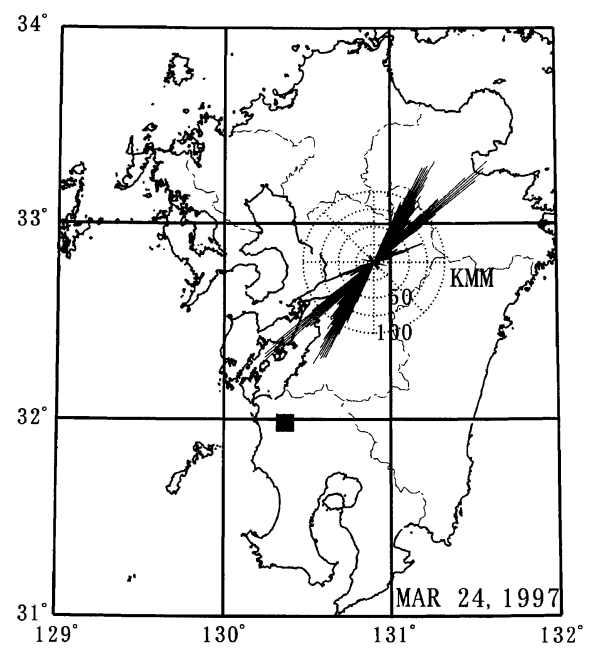

Fig. 7. Observation of VLF signals at Kumamoto on March 24, 1997 (Case II, an earthquake of $M=6.3$ on Mar. 26). The epicenter is shown with a black square.

the epicenter on a map. (iii) The number of occurrences of these emissions diminished towards Mar. 8 (d). (iv) On the day of the earthquake, Mar. 9, (e), the activity is still visible. (v) On Mar. 10 (f) there is little that suggests the presence of VLF activity related to the earthquake of the preceding day. Thus in the case of March 9, 1999 earthquake, the peak VLF emission occurred three days before it.

\subsection{Case IV: May 22, 1999 earthquake}

Results on the earthquake of May 22, 09:48 JST, 1999, $\mathrm{M}=4.1, \mathrm{~d}=23 \mathrm{~km}$, epicenter at $35.45^{\circ} \mathrm{N}, 139^{\circ} \mathrm{E}$ are presented in Fig. 10. The epicenter was close to Shonan Campus. Figure 10 shows observations at both the Shonan Campus and Shimizu stations. At Shonan Campus the VLF emission activity peaked on May 18, four days prior to the earthquake. The results shown in Fig. 10 are for May 18. The figure indicates that VLF emissions from the epicenter area were detected at both stations. The position of the VLF signal source area as determined by triangulation using the observations at the two stations is in gross agreement with the location of the epicenter. However, in part for the reasons discussed below, this should be considered to be a tentative deduction.

The features of VLF activity as observed at the two stations are shown differently in Fig. 11, in which the occurrence frequency of VLF signals received is plotted in the Cartesian coordinate representation with azimuth angles as abscisae. Results from Shonan Campus are shown on the right and those from Shimizu on the left side. The direction of the epicenter of the May 22 earthquake is indicated with an arrow. The azimuth angle is reckoned clockwise from the north; however, the ambiguity of $\pm 180^{\circ}$ in the azimuth angle should be kept in mind.

At Shonan Campus an activity peak sharply focussed at the direction of epicenter is clearly seen. This activity is a maximum on May 18, for which Fig. 10 is shown. A broader activity at Shonan Campus near $210^{\circ} \sim 250^{\circ}$ ( or $30^{\circ} \sim 70^{\circ}$ ) is considered to represent sferics activity. At Shimizu the VLF emission activity from the direction of the epicenter area and sferics activity are superimposed on each other, making it difficult to differentiate between the emissions from the two different sources. However, on May 18, the earthquake- 


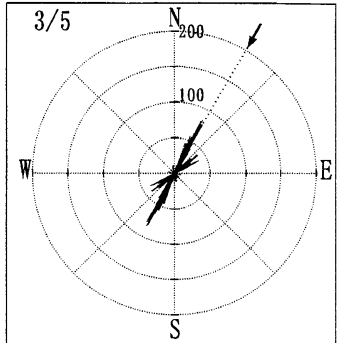

(a)

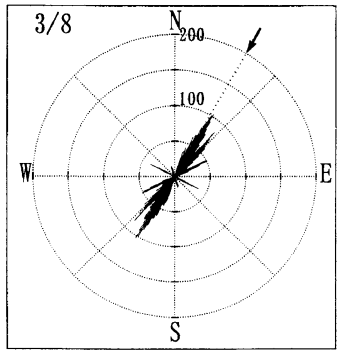

(d)

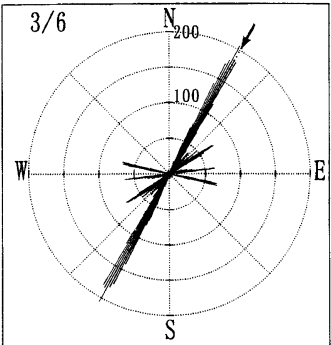

(b)

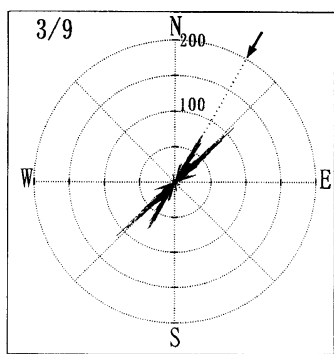

(e)

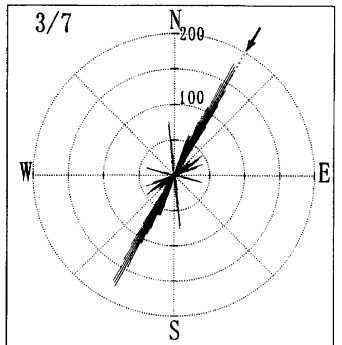

(c)

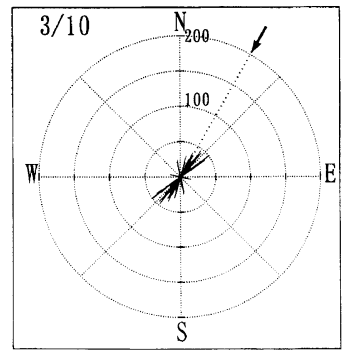

(f)

Fig. 8. The distribution of VLF signal arrival direction on each of six days, March 5 through 10, 1999 (Case III, an earthquake of M = 4.5 on Mar. 9). The epicenter direction is shown by a dotted line with an arrow at the tip.

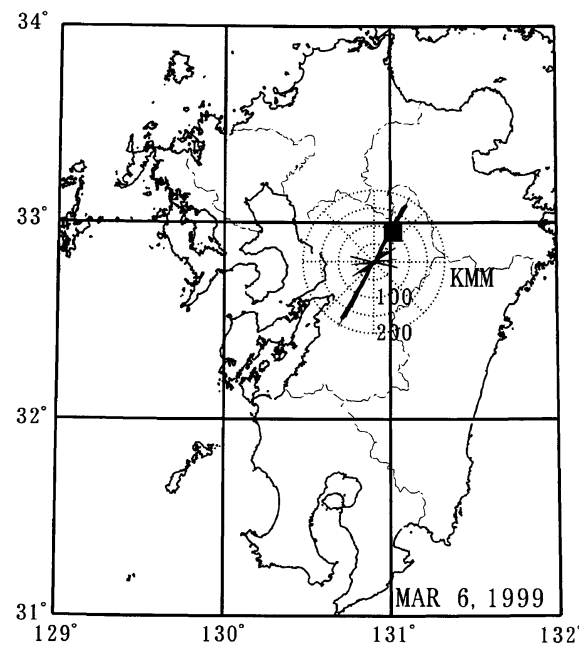

Fig. 9. Observation of VLF signals at Kumamoto on March 6, 1999 (Case III, an earthquake of $\mathrm{M}=4.5$ on Mar. 9). The epicenter is indicated with a black square.

related activity and sferics activity appear to be resolvable.

It is remarked here that at this stage of our investigation the accuracy with which the signal arrival direction can be determined is not precisely known. It is uncertain whether or not the slight difference between the azimuth angle of the peak VLF activity and the direction of the epicenter seen for Shimizu in Fig. 10 is real. Also we will have to keep in mind that the Shimizu station is close to the shore of the Sagami Bay so that coastal effects on the propagation of electromagnetic signals may not be negligible. Clarification of these details will have to await an accumulation of experience.

\section{Discussion}

We have presented four examples of observation of VLF

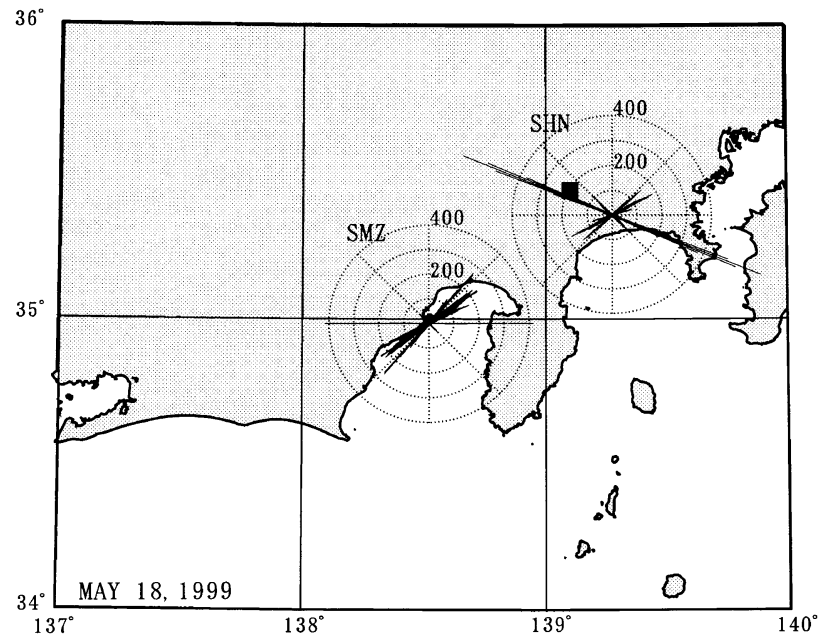

Fig. 10. Observation of VLF Signals at Shonan Campus and Shimizu (Shonan Campus being more to the east of Shimizu) on May 18, 1999 (Case IV, an earthquake of $\mathrm{M}=4.1$ on May 22). The epicenter is indicated with the black square.

activity that we consider as being related to earthquakes. The association of the VLF emissions with the occurrence of an earthquake is inferred from (a) temporal correlation and (b) agreement between the direction of VLF signal arrival and that of the epicenter. With the VLF signals that are subjected to our investigation, the occurrence frequency of the earthquake-related signals is found to be a maximum not on the day of the earthquake but a few days prior to it. With the four examples shown above, the maximum VLF activity was found 1 to 4 days before the day of the earthquake. Whether or not this time difference is related to the magnitude, depth, or any other parameter of the earthquake cannot be ascertained from the small sample we have at hand. Nor is it our 
(A)

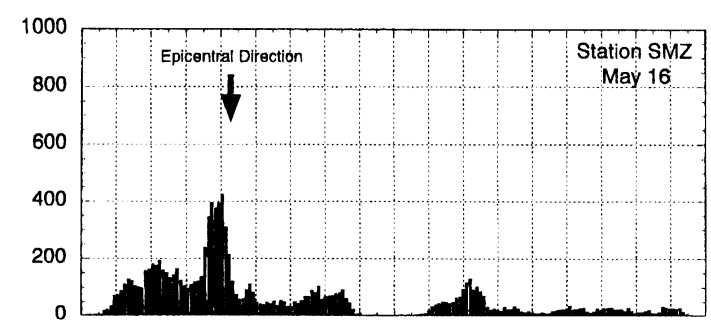

(B)

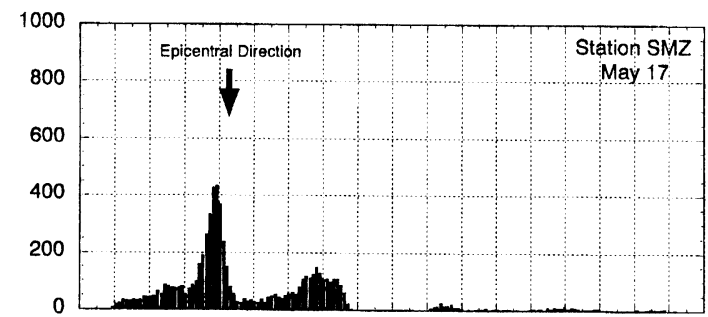

(C)

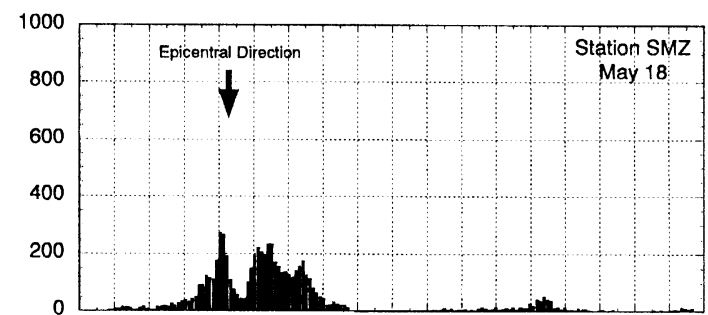

(D)

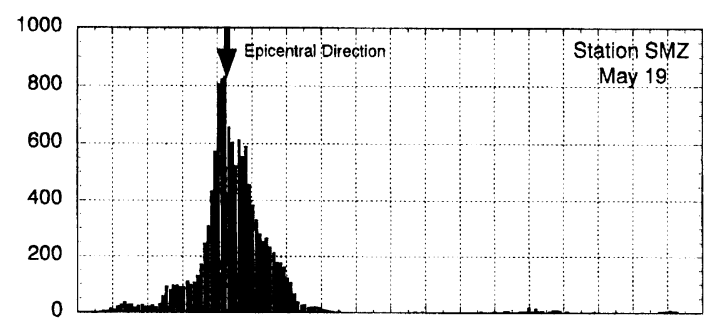

(E)

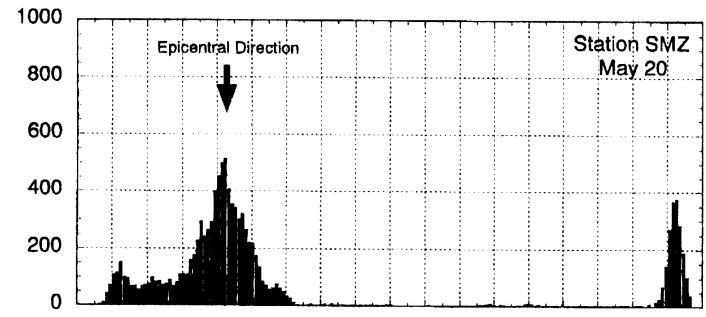

(F)

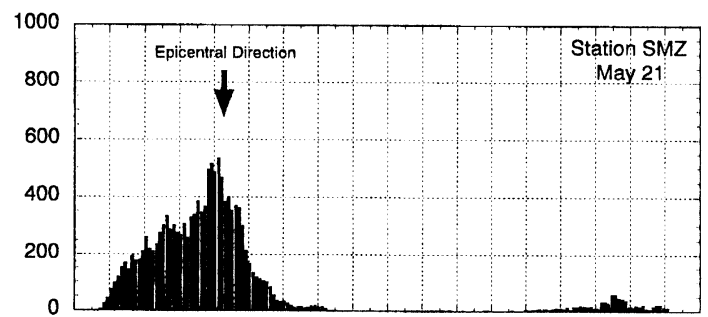

(G)

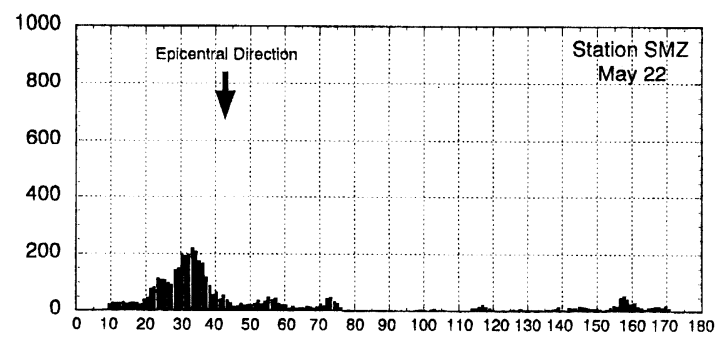

(a)

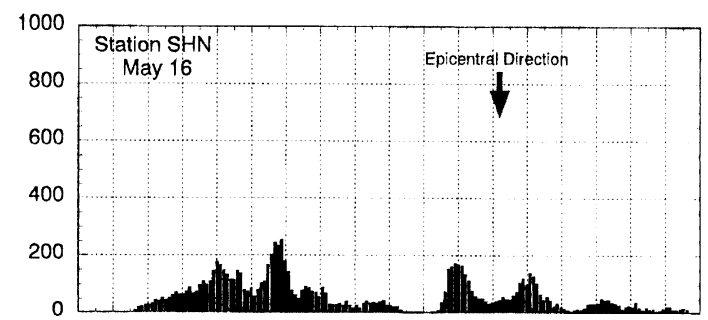

(b)

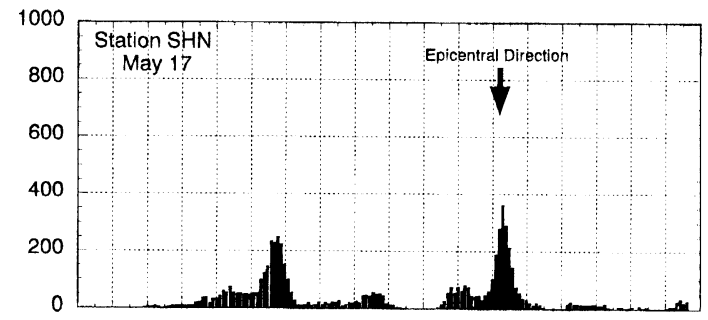

(c)

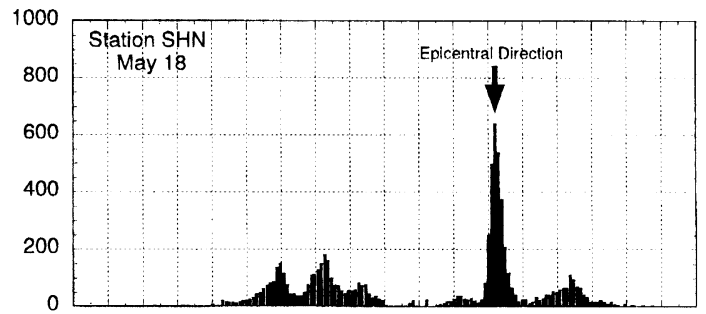

(d)

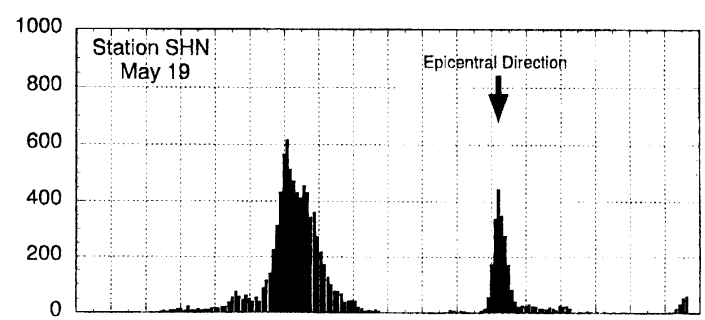

(e)

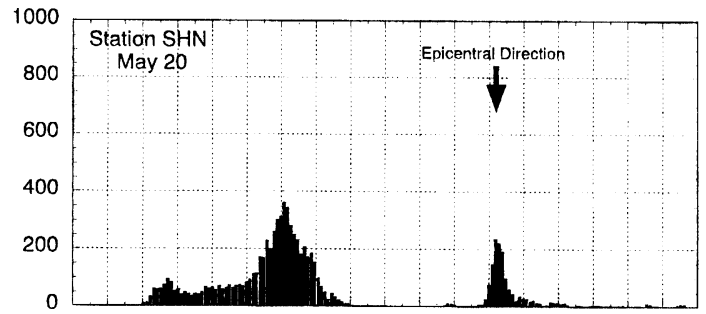

(f)
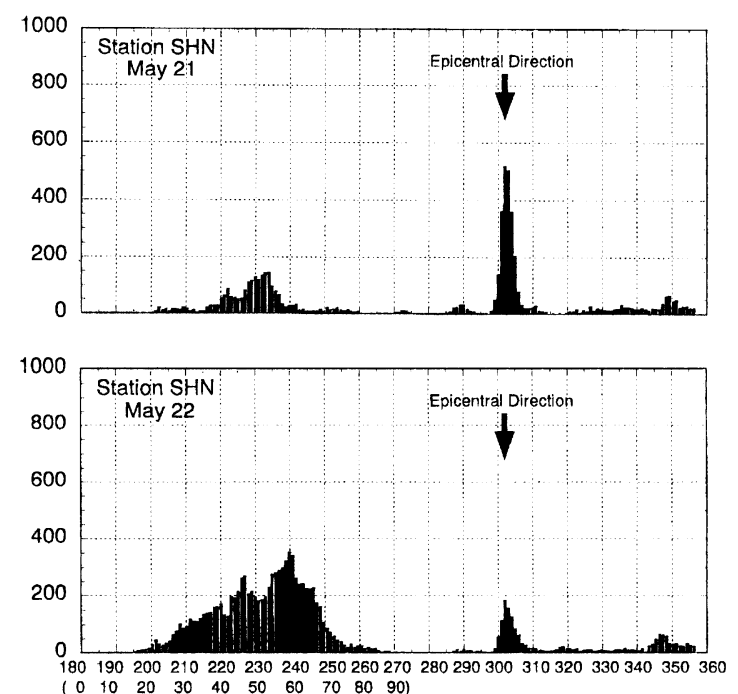

Fig. 11. The number of occurrence of VLF signals plotted against azimuth angle, for Shimizu (on the left) and Shonan Campus (on the right) both for May 16 through 22, 1999 (Case IV, an earthquake of $M=4.1$ on May 22) 
intention to discuss such details in this report. However, the fact that the time of the maximum VLF emission does not coincide with that of the main shock of the earthquake must be considered to constitute an important element in the VLF emission mechanism.

Warwick et al. (1982) published a valuable paper on an unusual radio emission at $18 \mathrm{MHz}$ observed on May 16, 1960, which was initially reported by Warwick (1963) in a context not related to seismological study. Upon re-examination of this radio event some twenty years later Warwick et al. (1982) concluded that there is enough reason for believing that the radio emission observed by Warwick in 1960 was related to the great Chilean earthquake of May 22, 1960. The radio event was observed by a network of radio receivers for cosmic radio noise at $18 \mathrm{MHz}$ that was intended to detect solar activity through the sudden decrease of $18 \mathrm{MHz}$ noise that extreme ultraviolet and $\mathrm{X}$ ray flare emissions induce in the earth's lower ionosphere. To support their contention Warwick et al. presented the results of a laboratory experiment that shows that electromagnetic emissions are produced when microfractures occur in Westerly granite. A similar laboratory study had been made by Nitsan (1977), in which he fractured quartz-bearing rocks and simultaneously recorded electromagnetic fields. Warwick et al. (1982) discussed in detail how their laboratory experiment relates to the radio emission observed in conjunction with the Chilean earthquake.

Since the frequencies of electromagnetic field variations are different between our observation and those made by Warwick et al., their analyses are not directly applicable to the phenomenon we are dealing with in this report. Nevertheless there is a considerable parallelism between the two different observations. There have been laboratory experiments in which electromagnetic signals from fractured rocks are in frequency ranges closer to the VLF range than the emissions dealt with in the laboratory experiments of Nitsan (1977) and Warwick et al. (1982). However, there is no physical ground to believe that such experiments are more relevant to our observation than the experiment quoted here. In any case, discussion of the emission mechanism for the VLF signals we observe in conjunction with earthquakes is outside the scope of this preliminary report, and will be deferred to future publication. Here we only hypothesize that the VLF emissions we have observed in relation to earthquakes are radiated by changes in polarization in the crystal structures of rocks caused by stress-induced fractures or similar processes that lead to the occurrence of earthquakes.

Lastly we add a remark on the range of the distance between the epicenter and the observation site for which our method is valid. This range cannot be precisely determined from the results thus far obtained. Obviously, at locations nearly directly above the source point of a VLF signal the horizontal direction of arrival of the signal cannot be unambiguously determined. Also, there must be an upper limit in the distance between the epicenter and the observing site beyond which the present technique becomes ineffective. Such an upper limit may vary from one region to another, and there may even be anisotropy in propagation in some areas. At this stage we have no other choice than taking up only those cases for which our technique is viable.

\section{Conclusions}

A technique has been developed to delineate VLF signals associated with earthquakes. The basic elements of our method include (i) selection of pulse-like VLF signals, (ii) temporal correlation with earthquakes, and (iii) selection of signals based on coincidence of the direction of signal arrival with that of the epicenter. (Details of selection (i) are given in Section 2.)

We have presented four examples of observation that, in our opinion, demonstrate a feasibility of delineating VLF emissions coming from the epicenter area 1 4 days preceding the earthquake which the VLF events are associated with.

With more experience and further refinements in the technique we hope to be able to open an approach towards earthquake prediction.

Acknowledgments. We acknowledge that the writing of this paper at this stage of our investigation was largely due to the urging of M. Otake and K. Tsumura. We wish to thank them for their encouragement. Thanks are also due to T. Nagao for his assistance in conducting this research. We are pleased to acknowledge valuable discussions with $\mathrm{S}$. Ono concerning the polarization in crystals. We acknowledge that comments by Y. Tanaka and another referee were helpful in improving the manner of presentation of the results. This paper is in part a dissertation of M. Kawazoe for the degree of Doctor of Science at the Tokai University.

\section{References}

Fujinawa, Y. and K. Takahashi, Emission of electromagnetic radiation preceding the Ito seismic swarm of 1989, Nature, 347(6291), 376-378, 1990.

Hayakawa, M. (ed.), Atmospheric and Ionospheric Electromagnetic Phenomena Associated with Earthquakes, 996 pp., Terra Scientific Publishing Company, Tokyo, 1999.

Hayakawa, M. and Y. Fujinawa (eds.), Electromagnetic Phenomena Related to Earthquake Prediction, Terra Scientific Publishing Company, Tokyo, 1994.

Maeda, K. and N. Tokimasa, Decametric radiation at the time of the Hyogoken Nanbu Earthquake near Kobe in 1995, Geophys. Res. Lett., 23, 2433 2436, 1996.

Nitsan, U., Electromagnetic emission accompanying fracture of quartzbearing rocks, Geophys. Res. Lett., 4, 333-336, 1977.

Warwick, J. W., Radio astronomical techniques for the study of planetary atmospheres, in Radio Astronomical and Satellite Studies of the Atmosphere, edited by J. Aarons, 400 pp., North Holland, Amsterdam, 1963.

Warwick, J. W., C. Stoker, and T. R. Meyer, Radio emission associated with rock fracture: Possible application to the great Chilean earthquake of May 22, 1960, J. Geophys. Res., 87(B4), 2851-2859, 1982.

Yoshino, T., I. Tomizawa, and T. Shibata, The possibility of using a direction finding technique to locate earthquake epicenters from electromagnetic precursor radiation, Ann. Geophys., 3, 727-730, 1985.

T. Asada, H. Baba (e-mail: hbaba@keyaki.cc.u-tokai.ac.jp), M Kawazoe, and M. Sugiura (e-mail: sugiura@jspan.kugi.kyoto-u.ac.jp) 